\title{
ZUR VERMEIDUNG VON THERMISCHEN EFFEKTEN BEI DER MESSUNG DER STRÖMUNGSDOPPELBRECHUNG
}

\author{
von $H$. JANESCHITZ-KRIEGL \\ Centraal Laboratorium T.N.O., Delft, Nederland.
}

\begin{abstract}
Synopsis
In order to avoid the thermal perturbation of flow birefringence measurements a modified Couette-apparatus was developed. In the usual apparatus the heat of friction generated in the liquid is removed by thermal conduction through the cylinder walls. As discussed by Björnståh18) a stationary distribution of temperature is established showing a maximum at the centre of the gap. Consequently the light used in the measurements is refracted and is partly reflected by the cylinder walls. This perturbation severely limits the measurement of small flow birefringences. It may be avoided by superimposing a slow motion of the liquid parallel to the axis on the main stream in the Couette-apparatus. This convection current removes an important part of the heat of friction. Its consequences were studied theoretically and experimentally. Essential for the method proposed here is the use of glass cylinders. Indeed, the high thermal conductivity of metal walls suppresses the effect of a slow convection stream.
\end{abstract}

\section{Zusammenfassung}

Es wird eine Methode gegeben, die zur Vermeidung des von Björnståhl ${ }^{8}$ ) beschriebenen Störeffektes bei der Messung der Strömungsdoppelbrechung führt. Wenn die in der Flüssigkeit erzeugte Reibungswärme durch die Zylinderwände (Metallwände) des Couette-Apparates abgeführt wird, erhält man nach Björnståhl über den Spaltquerschnitt eine stationäre Temperaturverteilung, deren Maximum in der Mitte des Spaltes liegt. Dadurch wird ein Teil des den Spalt durchlaufenden Lichtes zu den Zylinderwänden abgelenkt und erleidet Reflexion. Temperaturmaximum und Lichtablenkung können durch die Verwendung eines langsamen Konvektionsstromes parallel zur Zylinderachse fast völlig vermieden werden. Voraussetzung hierfür ist die Verwendung von Glaszylindern, da als Folge der grossen Wärmeleitfähigkeit von Metallzylindern der Einfluss einer langsamen Konvektionsströmung nicht zur Geltung kommen könnte.

I. Einleitung. Zur Messung der Strömungsdoppelbrechung bedient man sich in der Regel eines Couette-Apparates, der bekanntlich aus zwei koaxialen Zylindern besteht. Im Zwischenraum zwischen den Zylinderflächen - meist einfach Spalt genannt - befindet sich die Lösung, deren optisches Verhalten untersucht werden soll. Lässt man nun einen der beiden Zylinder um die gemeinsame Achse rotieren, dann erhält man in der Flüssigkeit eine Strömung, die mathematisch besonders einfach wird, wenn das Verhältnis der 
Spaltbreite zum Zylinderradius klein genug ist. Dann wird das Strömungsprofil nämlich linear mit konstantem Strömungsgradienten.

Wir werden hier nicht auf Entstehung und Deutung der Strömungsdoppelbrechung eingehen. Wichtig ist für uns nur, dass bei verdünnten makromolekularen Lösungen, besonders bei Lösungen von Fadenmolekülen, der auftretende Doppelbrechungseffekt sehr klein sein kann. Man muss mit Phasenunterschieden von weniger als $10^{-2}$ rechnen, was einem Unterschied der Brechungsindizes von weniger als $10^{-8}$ entspricht.

Wie schon erwähnt, muss man mit relativ engem Spalt arbeiten. Diese Forderung wird jedoch noch in stärkerem Masse dadurch bedingt, dass der kritische Strömungsgradient, bei dem trubulente Strömung einsetzt, stark von der Spaltbreite abhängig ist. Der kritische Strömungsgradient liegt natürlich umso höher, je enger der Spalt ist. Ausserdem ist natürlich für die Grösse des kritischen Gradienten noch massgebend, ob der Aussenzylinder oder der Innenzylinder des Aparates rotiert. Man wird nach Möglichkeit einen rotierenden Aussenzylinder verwenden, wie aus den ausführlichen Arbeiten von G. I. Taylor ${ }^{1}$ ) und aus dem Übersichtsartikel von H. G. Jerrard ${ }^{2}$ ) zu ersehen ist. Wie dem auch sei, so wird man doch stets trachten, die Spaltbreite möglichst klein zu halten.

Man darf bei diesen Überlegungen jedoch nicht auf die Wellennatur des Lichtes vergessen, mit dessen Hilfe man die Doppelbrechung zu bestimmen gedenkt. Der Lichtstrahl wird parallel mit der Zylinderachse durch den Spalt geschickt. (Vgl. O. Snellman $\left.{ }^{3}\right)$ ). Es tritt dabei in engen Spalten stets Beugung des Lichtes auf. Das Beugungsbild der Eintrittsstelle des Lichtes in den Spalt kann dann mit Hilfe einer Linse entworfen werden. Warum ist nun das Auftreten der Beugungserscheinung so unangenehm? Es stellt sich nämlich heraus, dass nur die nullte Ordnung des erwähnten Beugungsbildes von Licht erzeugt wird, welches auf dem Wege durch den Spalt keine Reflexion von den Zylinderwänden erlitten hat (H. Janeschitz-Kriegl $\left.{ }^{4}\right)$ ) Bekanntlich wird linear polarisiertes Licht bei der Reflexion an Metallwänden schwach elliptisch. Die dadurch vorgetäuschte Doppelbrechung liegt jedoch bereits in der Grössenordnung der meist sehr schwachen Strömungsdoppelbrechung selbst. Im Prinzip kann also erst nach Abschirmung der höheren Ordnungen die nullte Ordnung obigen Beugungsbildes zur Messung kleinster Werte der Strömungsdoppelbrechung herangezogen werden *).

Vom optischen Standpunkt gesehen wäre es günstiger, wenn man anstelle von Metallzylindern solche aus Glas verwenden würde, da bei diesen der durch Reflexion erzeugte Störeffekt um etwa eine Grössenordnung kleiner ist. (Vgl. auch H. Wayland ${ }^{6}$ ) und J. Leray ${ }^{7}$ )) Ausserdem ist die erreich-

*) R. Cerf ${ }^{5}$ ) benutzt eine anders geartete Aufstellung, die das Auftreten einer Beugungserscheinung im Spalt weitgehend unterdrückt. Es würde hier zu weit führen, näher darauf einzugehen. 
bare Oberflächenqualität bei Glas wesentlich höher als bei Metall, was für die Qualität des Beugungsbildes sehr wichtig ist.

Bevor wir jedoch unsere Diskussion über das Material der Zylinderwände fortsetzen können, müssen wir noch bedenken, dass in der strömenden Flüssigkeit Reibungswärme produziert wird. Wenn die Flüssigkeit auf konstanter Temperatur bleiben soll, dann muss die erzeugte Wärme abgeführt werden. Die Wärme-abfuhr kann nun im Prinzip auf zwei verschiedene Arten erfolgen, durch Wärmeleitung oder durch Wärmekonvektion.

Bis heute hat man sich beinahe ausschiesslich der ersten Möglichkeit bedient. Die Wärme wird dabei stets durch die Zylinderwände abgeleitet. Im stationären Zustand erhält man über den Querschnitt des Spaltes eine parabolische Temperaturverteilung mit dem Maximum in der Mitte. (Y. Björnståhl $\left.{ }^{8}\right)$ ). Da der Brechungsindex der Flüssigkeit mit steigender Temperatur abnimmt, ist der Lichtweg in der Mitte des Spaltes am kürzesten. Die strömende Flüssigkeit wirkt auf das Licht wie eine negative Zylinderlinse. Ein grosser Teil des Lichtes, das bei gleichmässiger Temperaturverteilung den Spalt durchlaufen würde, ohne die Wände zu berühren, wird dadurch gegen die Wände abgelenkt und erleidet Reflexion. (Y. Björnståhl ${ }^{8}$ ), O. Snellman $\left.{ }^{3}\right)$ ).

Auf das genannte Beugungsbild wirkt sich die parabolische Temperaturverteilung tatsächlich so aus, als hätte man zwischen Spalt und abbildende positive Linse eine negative Linse eingeschaltet. Die nullte Ordnung wird jedoch mit zunehmendem Strömungsgradienten nicht nur kleiner, sondern verliert auch sehr rasch an Intensität. Darüber verwundert man sich nicht mehr, wenn man zu beiden Seiten des Beugungsbildes breite, kontinuierliche Zonen reflektierten Lichtes entdeckt. Durch den Verlust an Intensität der nullten Ordnung wird in den meisten Fällen die Messung der Strömungsdoppelbrechung bereits bei Strömungsgradienten unmöglich, die weit unterhalb des kritischen Gradienten liegen, bei dem die Turbulenz einsetzt.

Die Möglichkeit der Beseitigung der unerwünschten parabolischen Temperaturverteilung mit Hilfe eines Konvektionsstromes haben erstmalig W. Kuhn, H. Oswald und H. Kuhn ${ }^{9}$ ) erkannt. Sie bauten einen Apparat mit rotierendem Innenzylinder. Den Durchmesser des Aussenzylinders wählten sie wesentlich grösser, sodass in dem entstehenden breiten Zwischenraum praktisch bei jeder Tourenzahl turbulente Strömung herrschte. Dadurch erhielten sie einen ausgezeichneten Wärmeaustausch. Nun setzten sie ein schmales Segment eines Zylinders als sogenannte Insel in den Zwischenraum. Zwischen Insel und Rotor wurde der Spalt jedoch so eng gewählt, dass sich dort laminare Strömung ausbilden konnte, deren Strömungsdoppelbrechung nun gemessen wurde. Die erzeugte Reibungswärme wurde also direkt mit der Flüssigkeit zusammen abgeführt. Bei dieser Konstruktion treten allerdings neue hydrodynamische Probleme auf, die hier nicht diskutiert werden sollen. (vgl. H. Janeschitz-Kriegl $\left.{ }^{4}\right)$ ). 
Wir wollen zu Betrachtungen am konzentrischen Zylinderapparat zurückkehren und untersuchen, ob man nicht mit Vorteil einen Konvektionsstrom parallel zur Zylinderachse anwenden könne. Die Herstellung eines solchen Konvektionsstromes geschieht mit Hilfe einer Pumpe, die einen Druckunterschied zwischen den Stirnflächen der Zylinder erzeugt. (Die entstehende überlagerte Poiseuilleströmung muss natürlich so schwach bleiben, dass der Doppelbrechungseffekt nicht beeinflusst wird). Für diese Art der Konvektion zeigt sich der Vorteil von Zylinderwänden aus Glas, da bei der hohen Wärmeleitfähigkeit von Metallwänden der Einfluss einer langsamen Konvektionsströmung nicht zur Geltung kommen könnte.

In diesem Aufsatz sollen nun Temperaturverteilungen über den Querschnitt des Spaltes berechnet werden. Als allgemeines Ergebnis kann vorweggenommen werden, dass der Temperaturunterschied zwischen Spaltmitte und Wand durch die vorgeschlagene Konvektionsströmung wesentlich vermindert werden kann. In vielen Fällen kann man ohne Beeinträchtigung der Strömungsdoppelbrechung sogar erreichen, dass die Temparatur in der Mitte des Spaltes niedriger wird als an der Wand, was eine Konzentrierung des Lichtbündels in der Mitte des Spaltes zur Folge haben würde. Da bereits das oben erwähnte Beugungsbild nicht mehr mathematisch erfassbar ist, müssen wir den Einfluss, den die berechneten Temperaturverteilungen auf die Lichtfortpflanzung ausüben, einer genaueren experimentellen Untersuchung unterziehen.

II. Aufstellung der Differentialgleichung und der Randbedingungen. Wir verwenden folgende vereinfachenden Annahmen: (vgl. H. C. Brinkman 10) 1. Da die Spaltbreite klein gegen die Radien der Zylinder ist; ersetzen wir die Zylinderwände für die Rechnung durch zwei planparallele Platten. Unsere $x$-Richtung falle mit dem Lot auf diese Platten zusammen. $x=0 \ldots$ Mitte des Spaltes.

2. Die $z$-Richtung wird parallel mit den Platten gewählt. Sie stellt die Richtung des Konvektionsstromes dar.

3. Der Strömungsgradient, der durch die Rotation eines der erwähnten Zylinder in der Lösung entsteht, kommt in unserem vereinfachten Bilde nur mehr dadurch zum Ausdruck, dass wir die pro Volumseinheit in der Flüssigkeit erzeugte Reibungswärme über das ganze Flüssigkeitsvolumen konstant annehmen. Diese Annahme schliesst ein, dass

4. die Viskosität der Flüssigkeit durch die auftretenden Temperaturunterschiede nicht wesentlich beeinflusst wird.

5. Wir vernachlässigen die zusätzlich durch den Konvektionsstrom erzeugte Reibungswärme. Da die Gradienten dieser Strömung klein gegen den Gradienten der Hauptströmung bleiben müssen, und da sich die Gradienten beider Strömungen pythagoreisch addieren, wird der Fehler unmerkbar, der mit dieser Vernachlässigung verknüpft ist. 
6. Die Wärmeleitung in der $z$-Richtung wird vernachlässigt, da die Temperaturgradienten in der Richtung des Konvektionsstromes äusserst klein sind, wenn man sie mit den Temperaturgradienten in der $x$-Richtung vergleicht. Ausserdem werden in der $z$-Richtung grosse Wärmemengen durch Konvektion transportiert.

Wir können also analog zu der von $\mathrm{H}$. C. Brinkman ${ }^{10}$ ) gegebenen Methode folgende Differentialgleichung aufstellen:

$$
\lambda_{l} \frac{\partial^{2} T}{\partial x^{2}}+\eta q^{2}-w \cdot \varrho \cdot v_{z}(x) \frac{\partial T}{\partial z}=0
$$

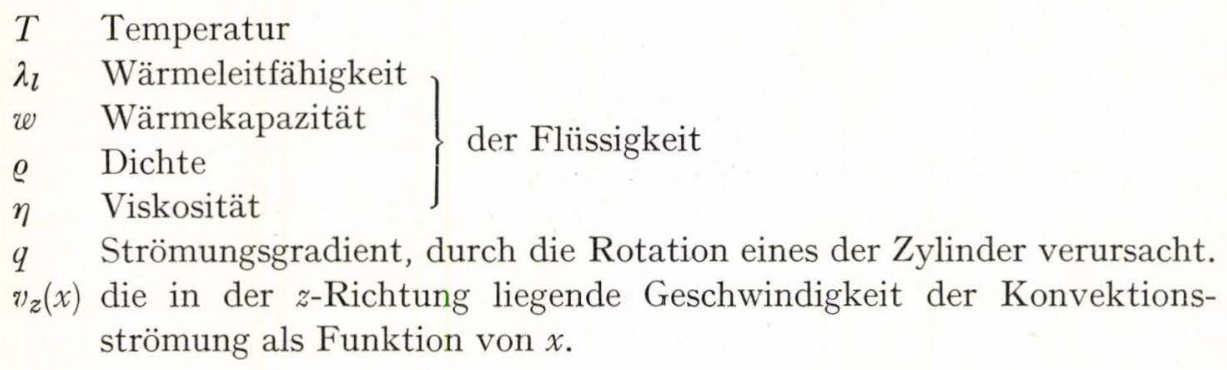

Der erste Term von Gl. (1) entspricht der Wärmemenge, die der Volumıseinheit in der Zeiteinheit durch Wärmeleitung in der $x$-Richtung zugeführt wird. Der zweite Term stellt die in der Zeiteinheit pro Volumseinheit erzeugte Reibungswärme dar. Der dritte Term beschreibt die durch den Konvektionsstrom transportierte Wärme. Im stationären Zustand muss natürlich die Summe dieser drei Wärmemengen in jedem Volumselement verschwinden. Diesen Umstand drückt Gl. (1) aus.

Für unseren Zweck genügt es, wenn wir annehmen, dass sich die untersuchte Lösung wie eine Newtonsche Flüssigkeit verhält. Für die Konvektionsströmung setzen wir daher die folgende Poiseuillesche Strömung:

$$
v_{z}=\frac{p}{2 \eta l}\left(\frac{d^{2}}{4}-x^{2}\right)
$$

p Druckdifferenz zwischen den Stirnflächen der Zylinder.

$l$ Höhe der Zylinder.

d Spaltbreite.

Bei Verwendung der dimensionslosen Variablen (vgl. H. C. Brin kman ${ }^{10}$ )

$$
\xi=\frac{2}{d} x, \quad \tau=\frac{\lambda_{l}}{\eta q^{2}} \frac{4}{d^{2}} T, \quad \zeta=\frac{\lambda_{l}}{w \cdot \varrho} \frac{16}{d^{3}} \frac{2 \eta l}{p \cdot d} z
$$

erhält man Gl. (1) in der folgenden Form:

$$
\frac{\partial^{2} \tau}{\partial \xi^{2}}+1-\left(1-\xi^{2}\right) \frac{\partial \tau}{\partial \zeta}=0
$$


An der Wand $(x=d / 2$ bzw. $\xi=1)$ gilt:

$$
\begin{aligned}
\left(T_{l}\right)_{w} & =\left(T_{g}\right)_{w} \\
\left(\lambda_{l} \frac{\partial T_{l}}{\partial x}\right)_{w} & =\left(\lambda_{g} \frac{\partial T_{g}}{\partial x}\right)_{w}
\end{aligned}
$$

$T_{l}$ Temperatur in der Flüssigkeit

$T_{g}$ Temperatur in der Glaswand

$\lambda_{g} \quad$ Wärmeleitfähigkeit des Glases

Da in Glas weder Reibungswärme erzeugt noch Wärme durch Konvektion abgeführt wird, gilt:

$$
\frac{\partial^{2} T_{g}}{\partial x^{2}}=0
$$

Die Wärmeleitung in der $z$-Richtung wird dabei (siehe 6. Vereinfachung) vernachlässigt. Als Folge von Gl. (6) wird die Temperaturverteilung in der Wand linear in $x$. Man erhält also

$$
\left(\frac{\partial T_{g}}{\partial x}\right)_{w}=-\frac{\left(T_{g}\right)_{w}}{D}
$$

$D$ Dicke der Wand.

Ohne Einschränkung der Allgemeinheit wurde die Temperatur an der Aussenseite der Wand konstant gleich Null gesetzt. Kombiniert man Gl. (7) mit den Randbedingungen (5), so erhält man für die Randbedingung von $\tau$ :

$$
\begin{gathered}
\left(\frac{\partial \tau}{\partial \xi}\right)_{\xi=1}+c \cdot \tau_{\xi=1}=0 \\
c=\frac{\lambda_{g}}{\lambda_{l}} \frac{d}{2 D}
\end{gathered}
$$

Da wir die Wandstärke zu beiden Seiten der Flüssigkeit gleich annehmen, gilt diese Randbedingung für $\xi= \pm 1$. Für die Randbedingung $z=0$ (Eintritt der Konvektionsströmung in den Spalt) ergibt sich ohne weiteres:

$$
\tau=0 \text { für } \zeta=0
$$

III. Lösung des Randwertproblems. Die Gleichungen (4), (8) und (10) können durch folgenden Lösungsansatz befriedigt werden: (vgl. auch R. B. Bird ${ }^{11)}$ )

$$
\tau=-\sum_{i=1}^{\infty} B_{i} X_{i}(\xi) \cdot e^{-a_{i} \xi}+\tau_{\infty}(\xi)
$$

Dabei stellt $\tau_{\infty}$ die Lösung in grossem Abstand vom Eintritt der Konvektionsströmung dar, wo ihr Einfluss auf die Temperaturverteilung bereits 
nicht mehr merkbar ist. Man erhält:

$$
\tau_{\infty}=\frac{1}{2}\left(1-\xi^{2}\right)+1 / c
$$

Die Funktionen $X_{i}(\xi)$ sind Lösungen der folgenden homogenen Differentialgleichung:

$$
X_{1}^{\prime \prime}+a_{i}\left(1-\xi^{2}\right) \cdot X_{i}=0
$$

Ihre Lösungen bilden ein vollständiges Orthogonalsystem mit reellen Eigenwerten $a_{i}$. Die Orthogonalitätsbedingung lautet:

$$
\int_{0}^{1}\left(1-\xi^{2}\right) X_{i} X_{k} \mathrm{~d} \xi=0 \quad i \neq k
$$

Da die $X_{i}$ für $\xi=0$ regulär und zum Ursprung symmetrisch sind, setzen wir folgende Reihenentwicklung an:

$$
X_{i}=\sum_{k=0}^{\infty} b_{i, k} \xi^{2 k}
$$

Einsetzen dieser Reihenentwicklung in die Diff.-Gl. liefert durch Koeffizientenvergleich die folgende Rekursionsformel für die $b_{i, k}$ :

$$
\begin{array}{r}
b_{i, k}=\frac{a_{i}}{2 k(2 k-1)}\left(b_{i, k-2}-b_{i, k-1}\right) \\
2 \leqslant k \leqslant \infty
\end{array}
$$

Wir können $b_{i, 0}=1$ setzen und erhalten dann:

$$
b_{i, 1}=-\frac{a_{i}}{2}, \quad b_{i, 2}=\frac{a_{i}}{12}+\frac{a_{i}^{2}}{24}
$$

Die Eigenwerte bestimmt man durch Einsetzen von (15) in die Randbedingung (8).

Man erhält:

$$
\sum_{k=0}^{\infty} b_{i, k}[2 k+c]=0
$$

Gl. (18) stellt ein Polynom in $a_{i}$ dar, das nur positive Wurzeln besitzt. Sie stellen nach ihrer Grösse geordnet die Eigenwerte unseres Problems dar.

Die Randbedingung (10) sieht unter Verwendung von (11) folgendermassen aus:

$$
0=-\sum_{j=0}^{\infty} B_{j} X_{j}(\xi)+\tau_{\infty}(\xi)
$$

Wegen der Orthogonalität der $X_{i}$ erhält man dann:

$$
B_{i}=+\frac{\int_{0}^{1} \tau_{\infty} \cdot X_{i} \cdot\left(1-\xi^{2}\right) \mathrm{d} \xi}{\int_{0}^{1} X_{i}^{2} \cdot\left(1-\xi^{2}\right) \mathrm{d} \xi}
$$

Damit ist die gestellte Aufgabe im Prinzip gelöst. Das Ergebnis hängt nur mehrvon den speziellen Werten der Konstanten $c$ ab, die wir in die Rechnung einsetzen müssen. 
IV. Berechnung der Temperaturverteilung in dimensionslosen Grössen. Die Randbedingung (8) bzw. (18) enthält neben dem allgemeinen Fall $0<c<\infty$ zwei Sonderfälle. $c=0$ stellt den Fall völliger Wärmeisolation dar, während $c=\infty$ bedeutet, dass $\tau_{\xi=1}=0$ sein muss. Letzterer Fall wird durch den Gebrauch dünner Metallwände approximiert. Glaswände sind bekanntlich relativ schlechte Wärmeleiter. So werden die von uns verwendeten Werte von $c$ im allgemeinen klein sein, $c$ hängt ausser von den Wärmeleitfähigkeiten auch noch vom Verhältnis der Spaltbreite zur Wanddicke ab. Da die numerischen Berechnungen einen grossen Arbeitsaufwand erfordern, wollen wir die Auswertung nur für zwei endliche Werte von $c$ ausführen. (Vgl. Gl. (9)).

Die im Laufe der Rechnung verwendeten Parameter wählen wir wie folgt:

TABELLE I

\begin{tabular}{rlrl|}
$\lambda_{g}$ & $=1,7 \cdot 10^{-3} \mathrm{cal} / \mathrm{cm}$. sec. grad. \\
$\lambda_{l}$ & $=5 \cdot 10^{-4}$ & & $\mathrm{cal} / \mathrm{cm}$. sec. grad. \\
& $=2,1 \cdot 10^{4}$ & & $\mathrm{erg} / \mathrm{cm} . \mathrm{sec}$. grad. \\
$w \cdot \varrho$ & $=0,5$ & & $\mathrm{cal} / \mathrm{cm}^{3} \cdot$ grad. \\
$\eta$ & $=10^{-2}$ & & erg sec. $/ \mathrm{cm}^{3}=10^{-2}$ poise \\
$D$ & $=1$ & & $\mathrm{~cm}$ \\
$d_{1}$ & $=2,5 \cdot 10^{-2}$ & $\mathrm{~cm}\left(=\frac{1}{4} \mathrm{~mm}\right)$ \\
$d_{2}$ & $=1 \cdot 10^{-1}$ & & $\mathrm{~cm}(=1 \mathrm{~mm})$ \\
$l$ & $=5$ & & $\mathrm{~cm}$ \\
\hline
\end{tabular}

Der Zusammenfassung halber haben wir in Tabelle (1) auch Parameter $(l, w . \varrho, \eta)$ aufgenommen, die wir erst später benötigen.

Die thermischen Konstanten der meisten organischen Lösungsmittel kommen überein mit den in der Tabelle (1) gegebenen Werten. Nur für Wasser liegen die Verhältnisse etwas anders. Es lohnt sich jedoch nicht, näher darauf einzugehen, da die in der Einleitung erwähnten optischen Effekte bei Wasser wegen der geringen Abhängigkeit seines Brechungsindex von der Temperatur viel weniger ausgeprägt sind. (Für org. Lösungsmittel gilt bei Zimmertemperatur ungefähr:

$$
\left.\frac{\mathrm{d} n}{\mathrm{~d} t}=5 \cdot 10^{-4}, \text { für Wasser } \frac{\mathrm{d} n}{\mathrm{~d} t}=1 \cdot 10^{-4}\right) .
$$

Da wir alle übrigen Parameter konstant halten, bekommen wir für die beiden in Tabelle (1) angeführten Spaltbreiten die folgenden Werte für die Konstante c: (vlg. Gl. 9)

$$
\begin{array}{ll}
c_{1}=0,043 & d_{1}=2,5 \cdot 10^{-2} \mathrm{~cm} \\
c_{2}=0,17 & d_{2}=1 \cdot 10^{-1} \mathrm{~cm}
\end{array}
$$

Die Dicke der Glaswände setzen wir in beiden Fällen gleich $1 \mathrm{~cm}$.

In Tabelle (2) sind nun die errechneten Eigenwerte (siehe Gl. (18)) und die dazugehörigen Werte $B_{i}$ angegeben. Die Genauigkeit der Werte ist den jeweiligen Forderungen angepasst. 
TABELLE II

\begin{tabular}{|l|c|c|c|c|}
\hline \multicolumn{1}{|c|}{} & 0 & 0,043 & 0,17 & $\infty$ \\
\hline$a_{1}$ & 0 & 0,063178 & 0.23543 & 2,83 \\
$a_{2}$ & 18,4 & 18,6 & 19,0495 & 32,0 \\
$a_{3}$ & \pm 80 & \pm 80 & \pm 80 & $\ldots$ \\
$B_{1}$ & $\ldots$ & $+23,80$ & $+6,4202$ & $+0,63$ \\
$B_{2}$ & $\ldots$ & $-0,52$ & $-0,0456$ & $\ldots$ \\
$X_{1}(1)$ & $\ldots$ & $+0,973767$ & $+0,90378$ & 0 \\
$X_{2}(1)$ & $\ldots$ & $-1,2$ & $-1,2305$ & 0 \\
$1 / c$ & $\infty$ & $+23,26$ & $+5,8824$ & 0 \\
\hline
\end{tabular}

Wegen der willkürlichen Annahme $b_{i, 0}=1$ gilt, dass die der Berechnung der $B_{i}$ zu Grunde gelegten Eigenfunktionen (siehe Gl. (15) u. Gl. (20)) in der Mitte des Spaltes den Wert eins haben. Die Werte an der Wand $(\xi=1)$ sind in Tab. 2 angegeben $\left[X_{i}(1)\right]$. An diesen Werten sieht man, dass für die ersten Eigenfunktionen (kleine Werte von $c$ vorausgesetzt) scheinbar nur ein geringer Unterschied zwischen den Werten in der Mitte und an der Wand besteht. Dies ist jedoch nur scheinbar so, da wegen der Grösse der Werte von $B_{1}$ der absolute Unterschied der Funktion

$$
B_{1}\left[X_{1}(\xi)-X_{1}(1)\right]
$$

in der Grössenordnung von 0,5 liegt, wie dies auch für $\tau_{\infty}$ gilt (vgl. Gl. (12)) Der Einfluss der ersten Eigenfunktion auf die Temperaturverteilung im Spalt ist daher entscheidend. Daneben sorgt die erste Eigenfunktion auch für die Beschreibung der Wandtemperatur.

Trennt man die Wandtemperatur in Gl. (11) ab, so erhält man:

$$
\begin{aligned}
\tau=- & B_{1}\left[X_{1}(\xi)-X_{1}(1)\right] e^{-a_{1} \xi}-B_{2}\left[X_{2}(\xi)-X_{1}(1)\right] e^{-a_{2} \xi}-\ldots+ \\
& +\frac{1}{2}\left(1-\xi^{2}\right)+\left\{1 / c-B_{1} X_{1}(1) e^{-a_{1} \xi}-B_{2} X_{2}(1) e^{-a_{2} \xi}-\ldots\right\}
\end{aligned}
$$

Denkt man sich den in geschweifter Klammer stehenden Ausdruck für die Wandtemperatur weg, dann beschreibt Gl. (23) mit genügender Genauigkeit die Temperaturverteilung im Spalt.

Für die Interpretation ist es nun sehr angenehm, dass der erste Term von Gl. (23) praktisch nur in der Exponentialfunktion von der Konstanten $c$ abhängig ist. Der Ausdruck (22) ist - mit anderen Worten - von $c$ praktisch unabhängig.

Fig. 1 zeigt nun für $c=0,17$ den Verlauf der ersten und der zweiten Eigenfunktion neben $\tau_{\infty}$. Nach vorhergehender Subtraktion der Funktionswerte an der Wand wurden die Kurven so normiert, dass ihr Wert in der Mitte des Spaltes gleich eins wurde.

Die Figuren $2 a, 2 b, 3$ und 4 geben einen Überblick über die Verteilung der reduzierten Temperatur $\tau$ für $c_{1}=0,043, c_{2}=0,17$ und für $c=\infty$. Die reduzierte Temperatur an der Wand wurde stets subtrahiert. Für negative Werte von $\xi$ verlaufen die Kurven symmetrisch. Je kleiner die Werte der 
Konstanten $c$ sind, desto grösser sind die Werte von $\zeta$ (vgl. Gl. (3)), bei denen die Wirkung der Konvektionsströmung noch bemerkbar ist. (vgl. Fig. $2 a$ mit Fig. 4).

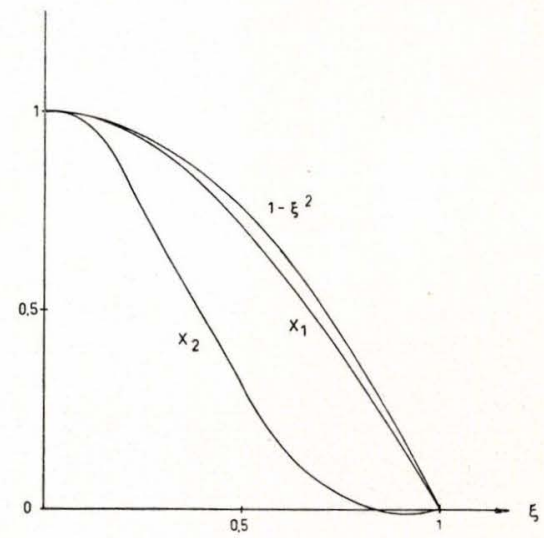

Fig. 1. Zum Formvergleich der Eigenfunktionen für den Parameter $c_{2}=0,17$.

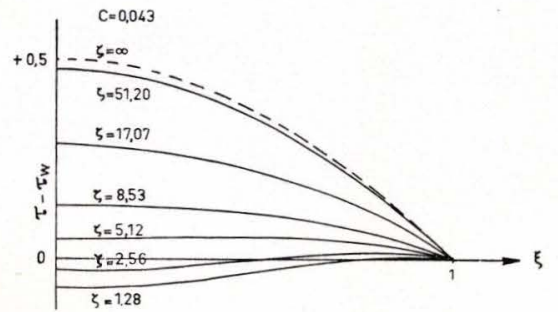

Fig. 2a. Verteilungskurven der reduzierten Temperatur $\tau$ für verschiedene reduzierte Abstände $\zeta$ von der Eintrittsstelle des Konvektionsstromes, wenn der Parameter $c_{1}=0,043$ ist. $\left(d_{1}=\frac{1}{4} \mathrm{~mm}\right.$, Glaswände) Für negative Werte von $\xi$ verlaufen die Kurven symmetrisch.

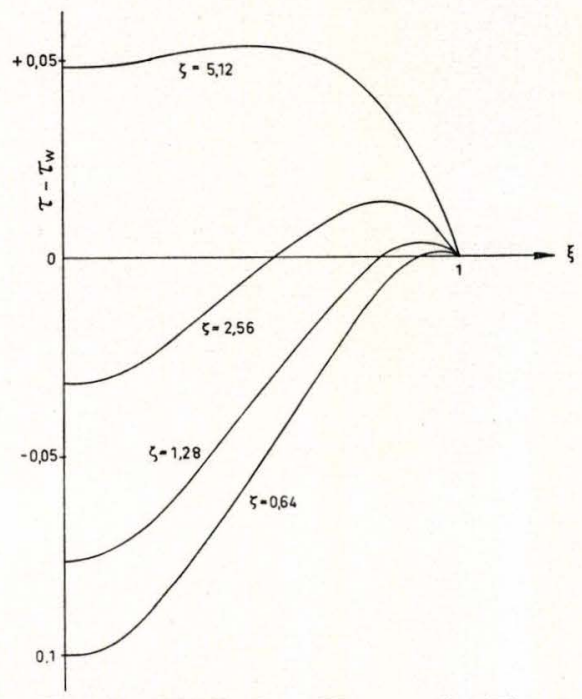

Fig. $2 b$. Die flachen Kurven der Fig. $2 a$ in grösserem Ordinatenmassstab.

$$
c_{1}=0,043 \text {. }
$$

V. Zulässiges Ausmass der Konvektionsströmung. In der reduzierten Differentialgl. (4) kommt das Ausmass der Konvektionsströmung nicht zum Ausdruck. Ihr Einfluss ist lediglich durch die dritte Transformationsgl. (3) gegeben. Setzen wir.

$$
\frac{p \cdot d}{2 \eta l}=q_{w}
$$


wobei $q_{w}$ den maximalen, an der Wand auftretenden Gradienten der Konvektionsströmung (Gl. (2)) darstellt, dann geht die dritte Gl. (3) über in:

$$
\zeta=\frac{\lambda_{l}}{w \cdot \varrho} \frac{16}{d^{3}} \frac{z}{q_{w}}
$$

$\zeta$ ist also einerseits proportional mit $z$, sodass man in den Figuren 2, 3 und 4 die Entwicklung des Temperaturprofiles mit zunehmendem $z$ verfolgen kann. Andererseits ist der starke Einfluss der Spaltbreite $d$ und der Konvektionsströmung auf den Proportionalitätsfaktor von z unverkennbar. Ersetzt man einen Spalt der Spaltbreite $d=\frac{1}{4} \mathrm{~mm}$ durch einen Spalt von $1 \mathrm{~mm}$, dann wird der genannte Faktor auf ein 64-stel reduziert. Beachtenswert ist, dass $\zeta$ nicht von den Eigenschaften der Zylinderwände abhängt.

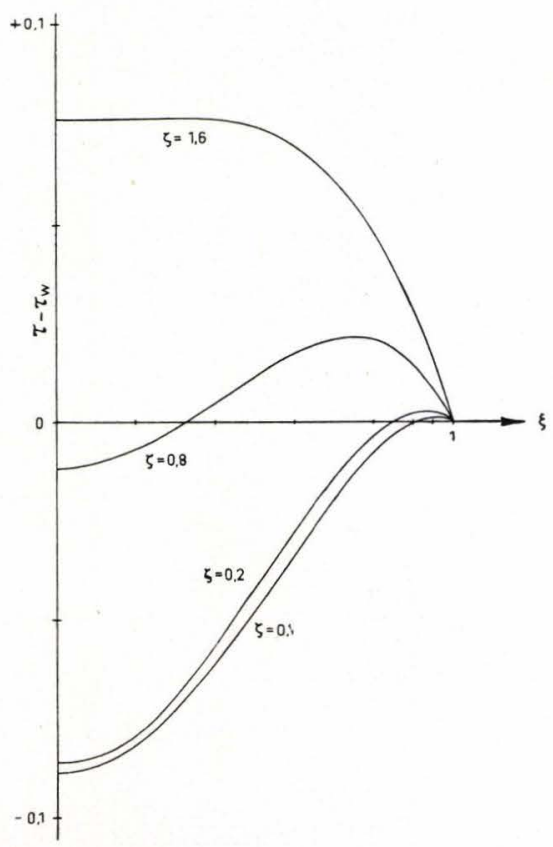

Fig. 3. Verteilungskurven wie in Fig. $2 b$, wenn der Parameter $c_{2}=0,17$ benützt wird. ( $d_{2}=1 \mathrm{~mm}$, Glaswände).

Wie man an Gl. (25) erkennt, wird man $q_{w}$ natürlich möglichst gross machen, damit $\zeta$ für den ganzen Bereich von $z[0 \leq z \leq l]$ klein bleibt.

Es bleibt also nur die Frage offen, wie gross man $q_{w}$ machen darf, ohne dass die durch den Gradienten $q$ der Couette-Strömung erzeugte Strömungsdoppelbrechung merkbar beeinflusst wird.

Man denke sich einerı doppelbrechenden, optisch zweiachsigen Kristall. Die Fortpflanzungsrichtung des polarisierten Lichtes falle mit einer der Hauptachsen des Indexellipsoides zusammen. Dann fallen die Auslöschungs- 
richtungen mit den beiden anderen Hauptachsen zusammen. Neigt man nun die zuerst genannte Achse einwenig gegen die ursprüngliche Fortpflanzungsrichtung des Lichtes, dann werden dadurch die Auslöschungsrichtungen in erster Näherung nicht geändert. Dasselbe gilt für den auftretenden Phasenunterschied.

Wir nehmen nun an, dass die durch die Konvektionsströmung erzengten Gradienten überall klein gegen den Gradienten der Couette-Strömung seien. Wegen der pythagoreischen Addition der Gradienten wird der Betrag des resultierenden Gradienten überall in erster Näherung mit dem Betrag des Gradienten der Couette-Strömung übereinstimmen. Es tritt daher in erster Näherung lediglich eine geringe, mit $x$ zunehmende Neigung derjenigen Achse des Indexellipsoides der Strömungsdoppelbrechung auf, die ohne Konvektionsstrom parallel zur Achse des Zylinderapparates und zur Lichtfortpflanzung stehen würde. Diese Neigung wirkt sich in erster Näherung wiederum nicht auf die Messung aus.

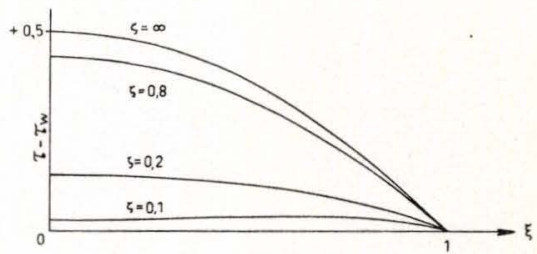

Fig. 4. Verteilungskurven wie in Fig. 2 und Fig. 3 wenn der Parameter $c=\infty$ benützt wird (dünne Metallwände), Ordinatenmassstab der Fig. $2 a$.

Setzen wir nun fest, dass der maximale, an der Wand auftretende Gradient der Konvektionsströmung ein Zehntel des Gradienten der Couette-Strömung ausmachen dürfe,

$$
q_{w}=\frac{q}{10}
$$

dann beträgt die beschriebene maximale Neigung an der Wand etwa 6 Grade, während der Betrag des Strömungsgradienten nur um ein halbes Prozent zugenommen hat. Wir erachten die dadurch auftretende Beeinflussung der Messergebnisse als völlig bedeutungslos, zumal die Effekte zu beiden Seiten der Spaltmitte einander entgegengesetzt sind. Bei völlig symmetrischer Ausleuchtung des Spaltes dürfte daher ein wesentlich stärkerer Konvektionsstrom keinen systematischen Fehler sondern lediglich eine Verminderung der Messgenauigkeit bewirken.

Es sei hier bloss erwähnt, dass die zulässige Grösse von $q_{w}$ im Prinzip auch noch durch einen anderen Umstand beschränkt wird. Die mit der Konvektionsströmung in den Spalt eintretende Flüssigkeit besitzt im allgemeinen keine Geschwindigkeitskomponente in der Richtung der CouetteStrömung. Zur Beschleunigung in dieser Richtung wird daher Energie ver- 
braucht, was eine Deformation des linearen Strömungsprofiles der CouetteStrömung zur Folge hat. Bei Erfüllung der Gl. (26) ist diese Deformation im Bereich der realisierbaren Strömungsgradienten $q$ auf alle Fälle unmessbar klein. Dies können wir aus Versuchen schliessen, die wir zur Prüfung der in der Einleitung erwähnten Konstruktion von W. Kuhn, H. Oswald und $\mathrm{H}$. Kuhn ${ }^{9}$ ) ausgeführt haben. (H. Janeschitz-Kriegl $\left.{ }^{4}\right)$ ).

VI. Berechnung der realen Temperaturverteilungen. Wir beschränken uns auf die Berechnung der Temperaturverteilung am Ende des Spaltes, wo der Konvektionsstrom den Zylinderapparat wieder verlässt. Dort treten die grössten Temperaturunterschiede auf. - Es ist daher vom Standpunkt des optischen Verhaltens günstig, die Lichtfortpflanzung in der Richtung des Konvektionsstromes und nicht in entgegengesetzter Richtung zu wählen. -

Setzt man in Gl. (25) $z=l$ und verwendet die Gl. (26), so erhält man für den Zusammenhang von $\zeta$ und $q$ :

$$
\zeta=160 \frac{\lambda_{l}}{w \cdot \varrho} \frac{l}{d^{3}} \cdot \frac{1}{q} .
$$

Mit den Werten der Tab. 1 erhält man:

$$
\begin{array}{ll}
d_{1}=\frac{1}{4} \mathrm{~mm} & q=\frac{5,12 \cdot 10^{4}}{\zeta} \mathrm{sec}^{-1} \\
d_{2}=1 \mathrm{~mm} & q=\frac{800}{\zeta} \mathrm{sec}^{-1}
\end{array}
$$

Aus der zweiten Gl. (3) erhält man unter Verwendung von Tab. 1:

$$
\begin{array}{ll}
d_{1}=\frac{1}{4} \mathrm{~mm} & T=7,44 \cdot 10^{-11} q^{2} \cdot \tau^{\circ} \mathrm{C} \\
d_{2}=1 \mathrm{~mm} & T=1,19 \cdot 10^{-9} q^{2} \cdot \tau^{\circ} \mathrm{C}
\end{array}
$$

In Fig. 2 kann man nun jedem $\zeta$ mit Hilfe von $(27 a)$ einen Strömungsgradienten $q$ der Couette-Strömung zuordnen. Gl. (28a) liefert dann die zu dem jeweiligen Strömungsgradienten gehörige Temperatur. Für Fig. 3 und Fig. 4 benötigen wir in derselben Weise die Gleichungen (27b) und (28b).

Die Figuren 5, 6 und 7 geben nun das Ergebnis dieser Transformation wieder. In den Figuren wurde nur die eine Hälfte des Spaltes, von der Spaltmitte bis zur rechten Wand gezeichnet. In der linken Spalthälfte verläuft die Temperaturverteilung symmetrisch. Fig. 5 zeigt Verteilungskurven für engen Spalt $\left(d_{1}=\frac{1}{4} \mathrm{~mm}\right)$ und Glaswand, Fig. 6 gibt die Verhältnisse bei weitem Spalt $\left(d_{2}=1 \mathrm{~mm}\right)$ und Glaswand, Fig. 7 bei weitem Spalt $\left(d_{2}\right)$ und Metallwand wieder.

Der Einfluss der Spaltbreite auf die Verteilungskurven ist nicht zu verkennen. Da der kritische Strömungsgradient, bei dem Turbulenz eirısetzt, beim weiten Spalt jedoch wesentlich niedriger ist, haben wir bei diesem entsprechend niedrigere Gradienten für die Darstellung verwendet. 
Beim weiten Spalt $\left(d_{2}=1 \mathrm{~mm}\right)$ erreicht man bei Anwendung von Gl. (26) wesentlich kleinere Werte von $\zeta$ als beim engen Spalt $\left(d_{1}=\frac{1}{4} \mathrm{~mm}\right)$. Dieser Umstand ist für die Brauchbarkeit der Methode bei weitem Spalt an und für sich schon sehr günstig. Aber selbst bei so günstigen $\zeta$-Werten tritt noch der Einfluss deutlich hervor, den das Material der Wände auf die Verteilung der Temperatur ausübt. Man vergleiche Fig. 6 und Fig. 7, wobei man die verschiedenen Massstäbe der Ordinaten beachte. Im Falle der Glaswände erscheint das Temperaturprofil praktisch eingeebnet. Bei höheren Gradienten tritt selbst ein neuer Effekt auf. Die Temperatur in der Mitte des Spaltes wird niedriger als an der Wand.

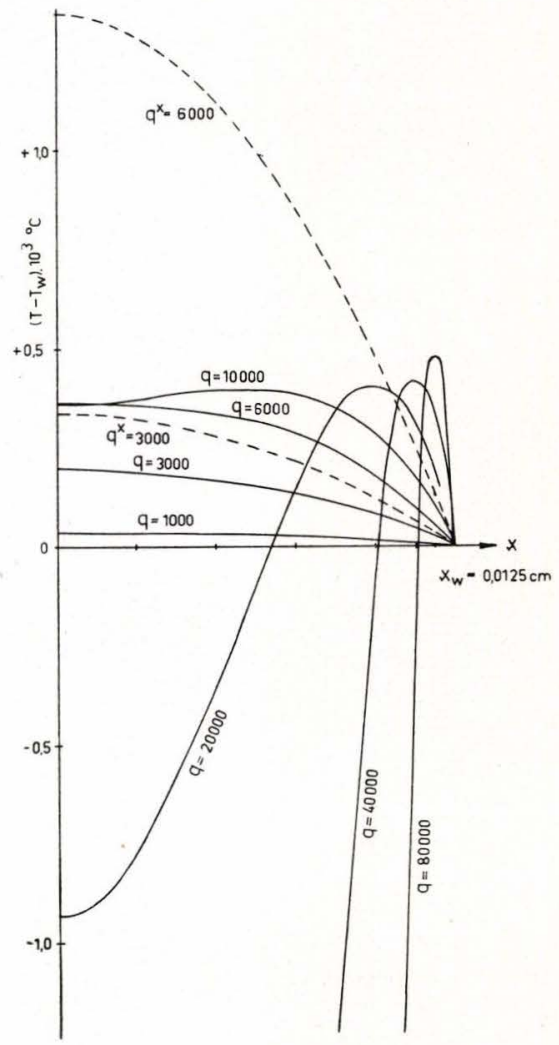

Fig. 5. Reale Temperaturverteilungen am Ende des Spaltes als Funktion der verwendeten Strömungsgradienten. Enger Spalt $d_{1}=\frac{1}{4} \mathrm{~mm}$, Glaswände. Die gestrichelten Kurven werden ohne Konvektionsstrom für die angegebenen Strömungsgradienten $q^{*}$ erhalten.

Zum Vergleich wurden in den Figuren gestrichelte Kurven eingezeichnet, die die Temperaturverteilungen zeigen, die ohne Konvektionsstrom erhalten werden. Man erhält diese Kurven mit Hilfe der Gleichungen (12) und (28). (mit $q^{*}$ versehen). 
Wie schon erwähnt, kann man beim engen Spalt $\left(d_{1}=\frac{1}{4} \mathrm{~mm}\right)$, wenn man an Gl. (26) festhalten will, keine so niedrigen Werte von $\zeta$ erhalten. Bei erster Betrachtung erscheint der enge Spalt daher für unsere Methode weniger günstig. Der Einfluss des Materiales der Wände wird hier jedoch entscheidend. Bei Verwendung von Metallwänden bewirkt ein durch Gl. (26) bemessener Konvektionsstrom keine erkennbare Abweichung von den in Fig. 5 eingetragenen gestrichelten Kurven, die ohne Konvektion erhalten werden. Die bei Verwendung von Glaswänden mit Konvektion erhaltenen Kurven sind hier jedoch sehr interessant. Einerseits versprechen die zu relativ niedrigen Strömungsgradienten gehörigen Kurven eine wesentliche Verminderung des in der Einleitung genannten optischen Störeffektes. Andrerseits wird bei hohen Gradienten eine gewisse Abschirmung der Wände gegen einfallendes Licht eintreten, die mit einer Konzentrierung des Lichtbündels verbunden sein muss. Man beachte in diesem Zusammenhang die Temperaturmaxima an den Wänden.

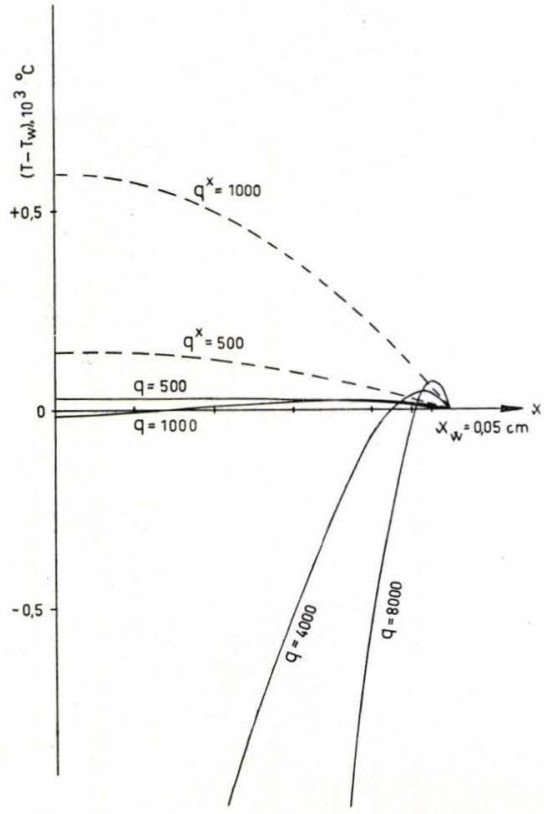

Fig. 6. Reale Temperaturverteilungen am Ende des Spaltes.

Weiter Spalt $d_{2}=1 \mathrm{~mm}$, Glaswände.

VII. Diskussion. Der Zweck der vorliegenden Untersuchung konzentrierte sich auf folgende Frage: Kann die Eindringtiefe eines langsamen Konvektionsstromes parallel zur Zylinderachse so gross gemacht werden, dass unter vernünftigen Bedingungen am Ende des Spaltes noch ein deutlicher Einfluss auf die Temperaturverteilung besteht? Dass dies möglich ist, wird durch die Grösse der Eigenwerte in Tab. 2 und durch die aus den 
Gleichungen (27) folgenden $\zeta$-Werte bestätigt. Alle Einzelheiten der gegebenen Verteilungskurven müssen jedoch vorsichtig interpretiert werden. Bis jetzt ist es nämlich noch nicht einmal gelungen, die Lichtfortpfanzung in einem engen Spalt zwischen reflektierenden Wänden exakt zu beschreiben, wenn der Brechungsindex des Zwischenmediums konstant ist.

Wir zogen daher die experimentelle Untersuchung vor, nachdem wir uns an Hand einfacher Überschlagsrechnungen davon überzeugt hatten, dass Hoffnung auf Erfolg bestand. In diesem Zusammenhang bedenke man, dass die in den Fig. 5 und 6 gegebenen Verteilungen am Ende des Spaltes auftreten. An der Eintrittsstelle des Konvektionsstromes herrschen dagegen voraussetzungsgemäss überhaupt keine Temperaturunterschiede. Also fällt der Vergleich mit den gestrichelten Kurven (Verteilungen ohne Konvektion) für das Konvektionsprinzip noch nicht günstig genug aus, da die gestrichelt gezeichneten Verteilungen in allen Querschnitten gleich ausgeprägt sind.

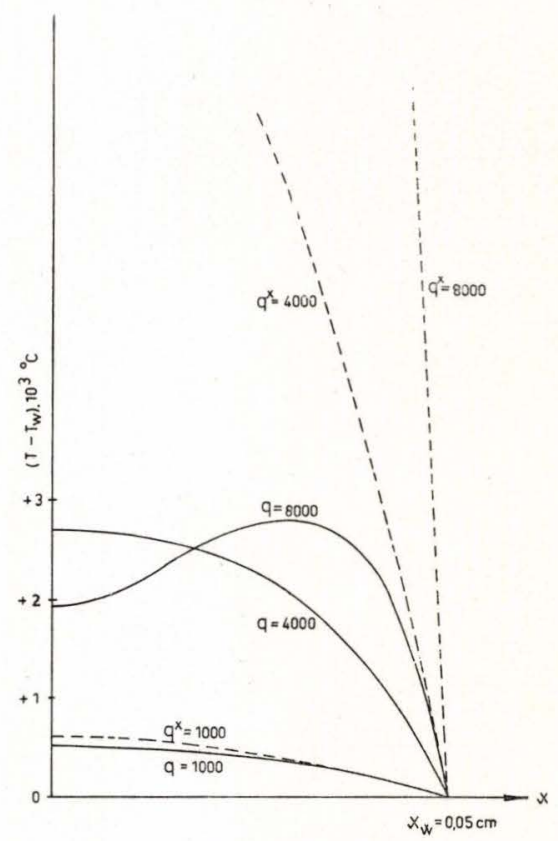

Fig. 7. Reale Temperaturverteilungen am Ende eines von Metallwänden gebildeten Spaltes der Breite $d_{2}=1 \mathrm{~mm}$. Beachte den gegen Fig. 5 und Fig. 6 verkleinerten Ordinatenmassstab.

Die konstruktiven Einzelheiten unseres Apparates sollen demnächst veröffentlicht werden. Auf jeden Fall arbeiten wir mit rotierendem Innenzylinder und einer Spaltbreite von $d=\frac{1}{4} \mathrm{~mm}$. Die kleine Spaltbreite sorgt dafür, dass der kritische Strömungsgradient relativ gross wird, bei dem Turbulenz einsetzt. Beide Zylinder sind aus schwarzem optischen Glas hergestellt und auf Hochglanz poliert. Das hier vorgeschlagene Konvektionsprinzip wird verwendet. Der verwendete enge Spalt versetzt uns in die Lage, 
die ausgeprägteren Temperaturverteilungen der Fig. 5 auf ihre optischen Auswirkungen $\mathrm{zu}$ untersuchen.

Das mit diesem Apparat erzielte experimentelle Ergebnis war, dass der in der Einleitung beschriebene von Björnståhl entdeckte Störeffekt praktisch nicht mehr auftrat. Bei Gebrauch einer relativ sehr viskösen Flüssigkeit $(\eta=10 \mathrm{ct}$ ps) vermeinten wir bei grossen Strömungsgradienten $(q=25000)$ selbst eine positive Linsenwirkung zu erkennen. Diese müsste davon stammen, dass dann die Temperatur in der Mitte des Spaltes niedriger wurde als an der Wand.

Das Übergangsgebiet von positivem zu negativem Temperaturunterschied zwischen Spaltmitte und Wand ist in Fig. 5 durch Gradienten zwischen 10.000 und 20.000 gekennzeichnet. Vermutlich reichen die in diesem Gebiet auftretenden Temperaturunterschiede jedoch noch nicht dazu aus, um die Fortpflanzungsrichtung des Lichtes merkbar zu beeinflussen. Bei der verwendeten viskösen Flüssigkeit konnten wir lediglich feststellen, dass die Einstellung des thermischen Gleichgewichtes ziemlich lange dauerte. Nach erreichtem Gleichgewicht unterschied sich jedoch das auftretende Beugungsbild praktisch nicht von dem im Ruhezustand erhaltenen Bilde.

In letzter Zeit wird die schlechte Wärmeübertragung durch eine Grenzflache (Flüssigkeit-Wand) oft erwähnt. Dem zu Folge wäre $c_{1}$ kleiner als in Gl. (2i) angegeben, was die Verschiebung des genannten Übergangsgebietes zu kleineren Strömungsgradienten verursachen würde. Da dann die auftretenden Temperaturunterschiede noch kleiner wären, könnte man im Übergangsgebiet noch weniger von einer befürchteten Störung der Lichtfortpflanzung bemerken.

Zum Schlıss seien noch einige Eigenschaften des vorgeschlagenen Apparates genannt. Die an der Wand auftretenden Temperaturen, durch G1. (23) (Ausdruck in geschweifter Klammer) gegeben, überschreiten praktisch nie die Grössenordnung von $0,1^{\circ} \mathrm{C}$. Nur in ganz extremen Fällen kann ein Temperaturanstieg um 1 bis $2^{\circ} \mathrm{C}$ stattfinden. Für einen Strömungsgradienten von $q=80.000$ erhielte man für $\eta=1$ ct ps eine Erhöhung um $1,06^{\circ} \mathrm{C}$. Dieselbe Erhöhung tritt bei einem $q=20.000$ auf, wenn die Viskosität $\eta=10$ ct ps beträgt.

Der durch Gl. (26) festgelegte Konvektionsstrom erfordert einen gewissen Flüssigkeitstransport. Das in der Zeiteinheit den Spaltquerschnitt passierende Flüssigkeitsvolumen ist durch folgende Gleichung gegeben: (Vgl. Gl. (2))

$$
\begin{array}{r}
V=\pi R \frac{d^{2}}{30} \cdot q=\pi R \frac{d^{3}}{6} \frac{p}{\eta \cdot l} \\
\text { R } \ldots \text { Radius des Rotors }
\end{array}
$$

Flüssigkeitsvolumen und Druckunterschied befinden sich darnach im experimentell leicht zugänglichen Gebiet. 
Schliesslich sei darauf verwiesen, dass der vorgeschlagene Apparattyp die Messung bei sehr hohen Strömungsgradienten ermöglicht. Es wird daher im Prinzip möglich sein, mit Hilfe einer von R. Cerf ${ }^{12}$ ) gegebenen Theorie die innere Viskosität von Fadenmolekülen bei grossen Strömungsgradienten zu untersuchen. Der Apparat ist jedoch ebenso gut zur Messung bei kleinen Strömungsgradienten geeignet.

Dem Direktor des "Centraal Laboratorium T.N.O.”, Dr. A. J. Staverman, sei für die Ermöglichung dieser Untersuchung und sein förderndes Interesse aufrichtig gedankt. Mein besonderer Dank gilt Herrn Prof. Dr. H. C. Brinkman für viele wertvolle Hinweise und Ratschläge.

Schliesslich sei die verlässliche Ausführung der numerischen Berechnungen durch Frl. E. van Meerten dankbar erwähnt.

Received 13-8-56.

\section{LITERATUR}

1) Taylor, G. I., Phil. Trans (A) 223, (1923) 289. Proc. roy. Soc. (London) A151 (1935) 494. A157 (1936) 546.

2) Jerrard, H. G., J. Applied Physics 21 (1950) 1007.

3) Snellman, O., Arkiv för Kemi, Min, Geol. 19A Nr. 30 (1945).

4) Janeschitz-Krieg1, H., Intern. Symp. Macromol. Chremistry Israel, April 1956. J. Polym. Sci. (in press).

5) Cerf, R., J. chim. physique $\mathbf{4 8}$ (1951) 85.

6) Wayland, H., J. phys. rad. 16 (1955) 27.

7) Leray, J., J. chim. physique $\mathbf{5 2}$ (1955) 755.

8) Björnståh1, Y., Z. Physik 119 (1912) 245.

9) Kuhn, W., Oswald, H. und Kuhn, H., Helv. chim. acta 36 (1953) 1209.

10) Brinkman, H. C., Appl. Sci. Res. Vol. A2 (1950) 120.

11) Bird, R. B., SPE Journal, Sept. 1955, p. 35.

12) Cerf, R., J. phys. rad. 15 (1954) 145. 